\title{
Peritoneal access - a pressing problem
}

\author{
Patrícia Valério $^{1}$ iD, Maria João Carvalho ${ }^{2}$ iD, Olívia Santos ${ }^{2}$ iD, António Cabrita ${ }^{2}$ iD, Anabela Rodrigues² iD \\ ${ }^{1}$ Nephrology Department, Centro Hospitalar de Setúbal; Setúbal, Portugal \\ 2 Nephrology Department, Centro Hospitalar Universitário do Porto, Porto, Portugal
}

\section{ABSTRACT}

In Portugal, as well as in Europe, peritoneal dialysis (PD) use remains low. Reorganization of PD units including a well-structured peritoneal access management protocol are fundamental to improve the take-up of this therapy. We analyzed the procedure and outcomes in a PD unit, addressing two key quality questions: at which level of estimated glomerular filtration rate (eGFR) were patients referred to peritoneal catheter placement and whether if it was timely placed.

We retrospectively evaluated all patients submitted to catheter placement between 2017 and June 2020. We analyzed the patient journey from Kidney Replacement Therapies (KRT) Option Appointment, until PD start, as well as demographic and clinical variables, including eGFR at four time points (KRT Options Appointment, PD unit evaluation, catheter placement, and PD start). To explore the adequacy of catheter placement schedule, we compared the characteristics of the patients who started PD within 90 days of catheter placement (Early group), and of those who started after the first 90 days (Late group).

We analyzed 48 patients in the Early and 27 in the Late group. The Early group presented a lower eGFR on KRT Options Appointment, with timely intervention: eGFR at catheter placement averaged $8.0 \pm 2.1 \mathrm{~mL} / \mathrm{min} / 1,72 \mathrm{~m}^{2}$. PD start occurred at $7.0 \pm 1.9$ and $8.0 \pm 2.4 \mathrm{~mL} / \mathrm{min} / 1.72 \mathrm{~m}^{2}$, in the Early and Late group, respectively. None of the patients suffered an urgent transition to HD by CVC. Four patients started PD less than 15 days after catheter placement, all of them without complications.

PD patients' admission involves specific tasks. Administrative tools or indicators to evaluate those processes are lacking. A peritoneal access clinic would allow the formalization of this circuit, allowing a quality and equitable approach to dialysis access. We suggest a structured pathway for peritoneal access management.

Keywords: peritoneal dialysis unit, peritoneal access management, quality improvement

(c) 2021 Portuguese Journal of Nephrology \& Hypertension. Published by Publicações Ciência \& Vida This is an open access article under the CC BY-NC-ND license (http://creativecommons.org/licenses/by-nc-nd/4.0/).

\section{INTRODUCTION}

In Portugal, as well as in Europe, peritoneal dialysis (PD) use remains low. ${ }^{1}$ This is a well-recognized problem among nephrologists, partially explained by structural and organizational problems of PD units. Adequate chronic kidney disease (CKD) education programs increase the odds of a patient's choice for PD. Also, creation of PD units and reorganization of existing ones, with dedicated health providers and facilities, are crucial. ${ }^{2,3}$

For any dialysis modality, the access is of primary significance. ${ }^{4,5}$ In our country, it is felt to be easier for physicians to start incident patients on hemodialysis (HD) rather than PD, since there is a well-established circuit from the pre-dialysis stage, including a vascular access (VA) appointment. Also, urgent start PD remains the exception, because of restraints related to peritoneal access. ${ }^{3}$

Nowadays, guidelines recommend education on all modalities of kidney replacement therapies (KRT) on CKD grade 4. This allows an early referral for dialysis access evaluation and subsequent creation, ideally when estimated glomerular filtration rate (eGFR) is between 15 to $20 \mathrm{~mL} / \mathrm{min} / 1.73 \mathrm{~m}^{2}$. When PD is the modality of choice, the catheter should be placed at least 2 weeks before the expected dialysis initiation. ${ }^{6}$

This leads us to question the level of peritoneal access management available in our country. There is no dedicated peritoneal access appointment or clinic, and the guidelines available only provide for the audit of the preoperative evaluation, catheter insertion technique and related complications 2,7 , lacking quality control of the pursuit between eligibility and final allocation to the home therapy.

We analyzed the procedure and outcomes in a PD unit in Portugal. This unit stands out for a high number of prevalent and incident DP patients, with about 26 to 28 admissions per year and around 100 prevalent patients in the last few years, with its PD penetration steadily increasing above $20 \%$. We aimed to address two key quality questions: at which level of GFR were patients referred to peritoneal catheter placement and whether if it was timely placed, meaning eviction of undesired central venous catheter (CVC) to start on HD. 


\section{SUBJECT AND METHODS}

We retrospectively evaluated all patients submitted to Tenckhoff catheter placement between 2017 and June 2020. Catheters are placed through mini-laparotomy with Moncrief-Popovich technique as an ambulatory procedure, except for the patients who need to start PD immediately (urgent start PD) or in cases of complex patients/ accesses.

All patients who chose PD were evaluated by PD medical and nursing team, to exclude potential contraindications and to clarify doubts. Ideally, a second assessment is performed when the patient is expected to start PD in the short term, allowing the catheter placement to be scheduled. Patients' circuit is heterogeneous, usually maintaining follow-up by the nephrologist until dialysis initiation. If the nephrologist is part of PD team, and for those referred by another hospital, the follow-up is maintained in the PD clinic during the pre-dialysis period.

The variables analyzed included eGFR at four time points (except for HD patients): KRT Options Appointment, PD unit evaluation, catheter placement, and PD start. We also collected demographic information and data on previous KRT and comorbidities. Patients were followed until August 2020 (two months after the last catheter placement).

We divided patients into 3 groups according to timing of PD start: the patients who started within 90 days of catheter placement (Early group), those who started after the first 90 days (Late group), and those who did not start PD during the follow-up (because of death, preemptive kidney transplantation or lost to follow-up). The
90 day period is the reasonable period considered by the unit to carry out the task-flow between catheter placement and PD start.

To explore the adequacy of catheter placement schedule, we compared the first 2 groups, assessing patients'characteristics for each one. The adequacy of catheter placement was defined according to guidelines ${ }^{2}$ as successful PD start, with a functional catheter, without complications related with catheter insertion (mechanical and infectious ones), specifically avoiding urgent need of HD with a CVC.

Categorical variables were expressed as absolute (n) and relative (\%) frequencies, and the chi-square statistic was used to assess the statistical significance between groups. Continuous variables were expressed as the mean \pm standard deviation or median and interquartile range (IQR). Normality was explored with the Shapiro-Wilk test. Then, parameters were compared by using a t-test if normally distributed or by Kruskal-Wallis/Mann- Whitney $U$ test if not normally distributed. A p-value less than 0.05 was considered statistically significant. All probabilities were two-tailed. The exploratory analysis and statistical modelling were performed using IBM $^{\circledR}$ SPSS $^{\circledR}$ version 23.0 (SPSS, Inc., Chicago, IL).

\section{RESULTS}

In the aforementioned period, 101 patients underwent catheter placement, three of them under the urgent-PD start program. Twenty-one (20.8\%) patients did not start PD during the follow-up, maintaining regular appointments at the nephrology clinic. We included in our analysis 48 patients in the Early group and 27 in the Late group (Figure 1). Twelve patients were already under HD.

Table I

Main characteristics of Early and Late groups.

\begin{tabular}{|c|c|c|c|c|c|}
\hline & \multicolumn{2}{|c|}{ Characteristics } & $\begin{array}{l}\text { Early group } \\
\mathrm{N}=48(\%)\end{array}$ & $\begin{array}{l}\text { Late group } \\
\mathrm{N}=27(\%)\end{array}$ & \\
\hline & \multicolumn{2}{|l|}{ Male gender, \% } & $31(64.6 \%)$ & $22(68.8 \%)$ & 0.811 \\
\hline & \multicolumn{2}{|c|}{ Mean age (on catheter placement), years } & $52.6 \pm 19.5$ & $56.8 \pm 12.2$ & 0.222 \\
\hline & \multicolumn{2}{|c|}{ Diabetic patients, $\%$} & $8(16.7 \%)$ & $12(37.5 \%)$ & 0.063 \\
\hline & \multicolumn{2}{|c|}{ Median age-adjusted Charlson Comorbidity Index } & 5 (IQR 4) & 6 (IQR 2) & 0.886 \\
\hline & \multicolumn{2}{|c|}{ Main kidney disease etiology - glomerular } & $21(43.8 \%)$ & $8(25.0 \%)$ & 0.102 \\
\hline \multirow[t]{2}{*}{ 1) KRT Options Appointment } & \multicolumn{2}{|c|}{ Mean eGFR, $\mathrm{mL} / \mathrm{min} / 1.72 \mathrm{~m}^{2} *$} & $13.3 \pm 5.4$ & $15.7 \pm 4.7$ & 0.014 \\
\hline & \multicolumn{2}{|c|}{ PD choice, due to autologous vascular access complexity/ failure } & $6(12.5 \%)$ & $1(3.1 \%))$ & 0.233 \\
\hline \multirow[t]{3}{*}{ 2) Assessment by PD team } & \multicolumn{2}{|c|}{ Median time since KRT Options Appointment, days } & $52.0($ IQR 164.0$)$ & 39.5 (IQR 246.8) & 0.892 \\
\hline & \multicolumn{2}{|c|}{ Mean eGFR, $\mathrm{mL} / \mathrm{min} / 1,72 \mathrm{~m}^{2} *$} & $12,6 \pm 6,4$ & $14,1 \pm 3,7$ & 0.344 \\
\hline & \multicolumn{2}{|c|}{ Follow-up with PD team/ Unit, \% } & $27(56.3 \%)$ & $14(43.8 \%)$ & 0.362 \\
\hline \multirow[t]{5}{*}{ 4) Catheter placement } & \multicolumn{2}{|c|}{ Median time since the first evaluation by PD team, days } & $23.0($ IQR 161.0) & 83.5 (IQR 262.0) & 0.040 \\
\hline & \multicolumn{2}{|c|}{ Mean eGFR, $\mathrm{mL} / \mathrm{min} / 1.72 \mathrm{~m}^{2} *$} & $8.0 \pm 2.1$ & $12.0 \pm 3.5$ & 0.001 \\
\hline & \multirow[t]{3}{*}{ Previous KRT } & None & $29(60.4 \%)$ & $27(84.4 \%)$ & 0.026 \\
\hline & & HD & $11(22.9 \%)$ & $1(3.1 \%)$ & 0.023 \\
\hline & & Kidney transplantation & $8(16.7 \%)$ & $4(12.5 \%)$ & 0.754 \\
\hline \multirow[t]{2}{*}{ 5) PD start } & \multicolumn{2}{|c|}{ Median time since catheter placement, days } & 39.0 (IQR 28.0) & 245.5 (IQR 163.5) & $<0.001$ \\
\hline & \multicolumn{2}{|c|}{ Mean eGFR, $\mathrm{mL} / \mathrm{min} / 1.72 \mathrm{~m}^{2} *$} & $7.0 \pm 1.9$ & $8.0 \pm 2.4$ & 0.914 \\
\hline
\end{tabular}

*For the patients already on HD, eGFR was not considered. Abbreviations: eGFR, estimated glomerular filtration rate; HD, hemodialysis; IQR, interquartile range; KRT, kidney replacement therapies; PD, peritoneal dialysis. 


\section{Figure 1}

Strobe diagram of patients included.

\section{1 patients submited to catheter placement}

\section{(2017 and June 2020)}

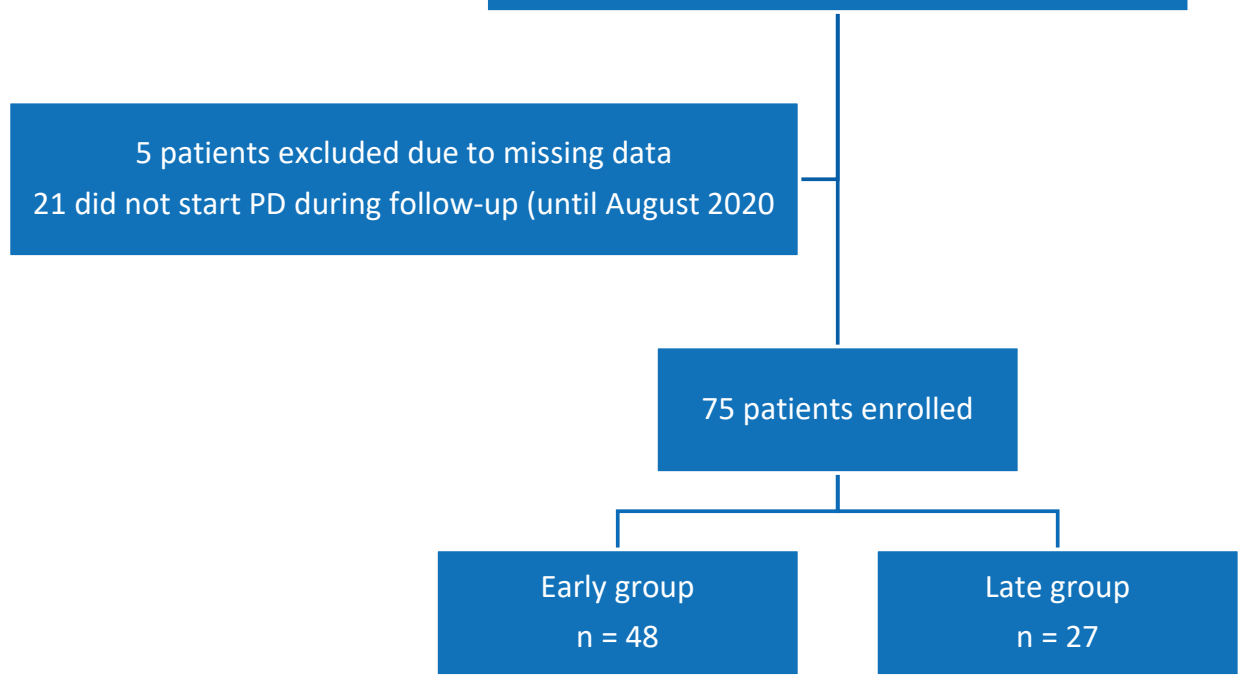

The majority ( $n=11)$ were in the Early group, usually in situations of great VA complexity. Table I presents the comparison between the 2 groups, with clinically relevant variables.

The Early group was presented to KRT Options Appointment with a lower eGFR, documenting appropriate circuit until PD induction at an eGFR of $7.0 \pm 1.9 \mathrm{~mL} / \mathrm{min} / 1.72 \mathrm{~m}^{2}$.

Considering the first assessment by the PD unit, the median time (days) since KRT Options Appointment did not differ significantly between the groups: the wide range in time (interquartile range- IQR) is related to early referral to KRT Options Appointment in certain cases, especially from other hospitals, leading to an appropriate postponed appointment by the PD unit. An additional reason is related to the intermediate evaluation after KRT Options Appointment, by the nephrologist, who requests the PD unit to be scheduled according to how urgent they feel the case is.

In the Early group, four patients started PD less than 15 days after catheter placement, all of them without complications related with the procedure: 3 of them under the urgent PD-start program; the remaining one on the $14^{\text {th }}$ day, without complications. All patients successfully started PD with functional catheters. None of the patients transitioned to urgent HD with a CVC.

\section{DISCUSSION}

The process of PD patients' admission is complex, involving specific tasks: 1) evaluation of conditions for PD; 2) option, validation, and consent for catheter placement; 3) preoperative evaluation; 4) scheduling catheter placement; 5) evaluation after catheter placement; 6) planning of PD start; 7) assessment and management of peritoneal access complications in transition processes (from /to HD, from /to kidney transplantation) in patients who are not active in the PD program. Despite the guidelines available to audit the preoperative evaluation and catheter placement, administrative tools and quality indicators are lacking for the remaining part of the process.

A peritoneal access clinic would allow the formalization of this circuit within a broader mapping of PD allocation pathway, that includes the processes of: 1) option; 2) acess; and 3) PD induction. This should be a requirement for any nephrology department, in parallel with a VA appointment, because it represents the recognition of the complexity of all pre-dialysis patients and a step towards higher quality of services. This strategy will allow us to identify bottlenecks in the patient circuit, promote solutions and allocate resources.

Presently, this PD unit overcomes organizational insufficiencies with dedicated and resilient staff, but diagnosis must be made: there is und efined structure and capacity, activities remain formally unmanaged (unlike with VA), and specific quality indicators are lacking, to promote improvement. In spite of that, a clinically efficient catheter placement circuit was achieved. The extent time period until catheter placement depends on the clinical judgment and priority given by the PD team, assuring a rapid response for patients referred later or with an urgent need for PD. In some patients, with slow kidney disease progression, the catheter placement was appropriately postponed. In fact, the period between nephrologist referral and catheter placement would be the best indicator to evaluate the PD clinic response 


\section{Figure 2}

Pathway suggested for management of peritoneal access.

\section{Management of Peritoneal Access}

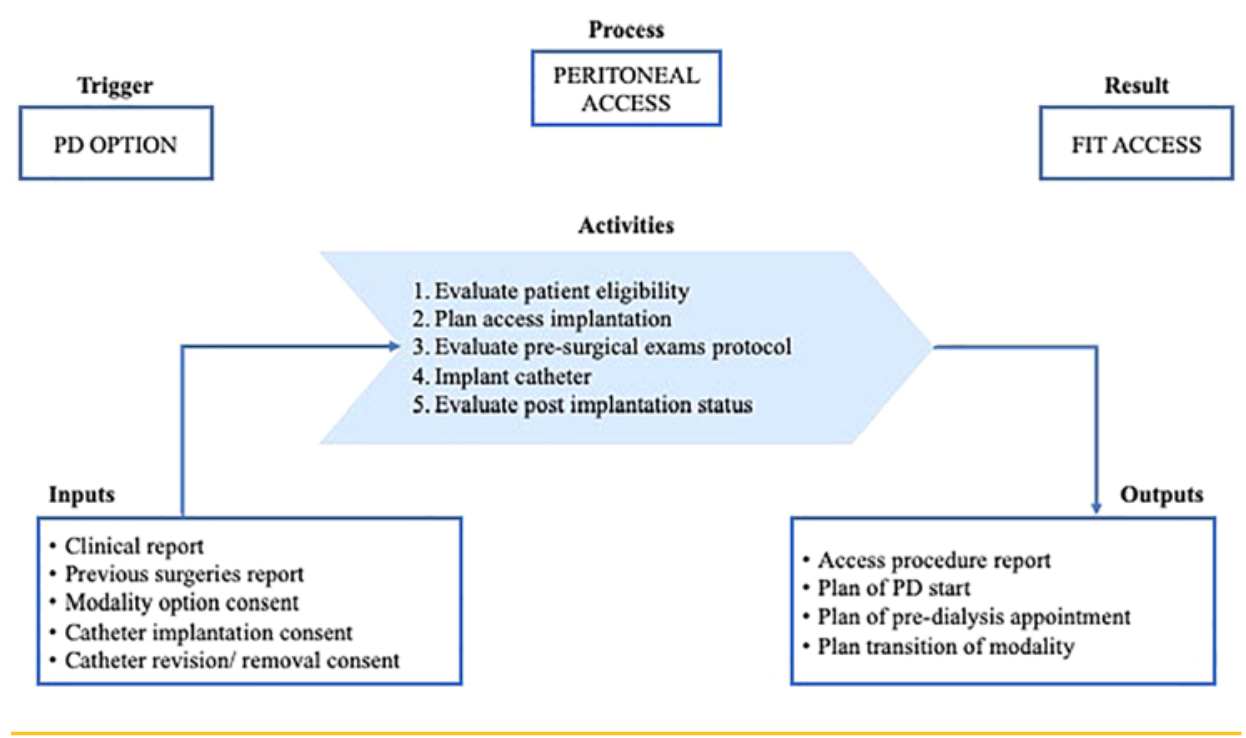

time, with potential for its use as a quality indicator. The percentage of CVC used in dialysis induction of patients who elected PD is an added quality indicator and null is the objective.

In conclusion, a peritoneal access clinic would allow the formalization of pre-PD circuit, and it should be a requirement for any nephrology department, towards a quality and equitable approach to dialysis access. Figure 2 suggests a structured pathway of activities for peritoneal access management, including pre-operative checklist, access placement and quality indicators. Benchmarking and peer training are some feasible tracks, only dependent on institutional strategy and effective patient centered nephrology.

Disclosure of potential conflicts of interest: none declared.

\section{References}

1. Society PN. Portuguese Registry of Dialysis and Transplantation 2019. http://www.bbg01.com/ $\mathrm{cdn} /$ rsc/spnefro/gabreg/310/ER2020_Registo.pdf; 2019.

2. Figueiredo A, Goh BL, Jenkins S, Johnson DW, et al. Clinical Practice Guidelines for Peritoneal Access. Perit Dial Int 2010:30(4):424-9.

3. Finkelstein FO. Structural Requirements for a Successful Chronic Peritoneal Dialysis Program. Kidney Int Suppl 2006(103):S118-21.
4. Crabtree JH, Shrestha BM, Chow KM, Figueiredo AE, et al. Creating and Maintaining Optimal Peritoneal Dialysis Access in the Adult Patient: 2019 Update. Perit Dial Int 2019;39(5):414-36.

5. Gardezi Al, Sequeira A, Narayan R. Going Home: Access for Home Modalities. Adv Chronic Kidney Dis 2020;27(3):253-62.

6. Group. KDIGOKCW. Kdigo 2012 Clinical Practice Guideline for the Evaluation and Management of Chronic Kidney Disease. Kidney Inter 2013(3):1-150.

7. Manual de Boas Práticas de Diálise Crónica da Ordem dos Médicos, (2017).

\section{ORCID}

Patrícia Valério (iD) 0000-0001-7397-0233

Maria João Carvalho (iD) 0000-0003-4374-2867

Olívia Santos (iD) 0000-0002-0193-3625

António Cabrita (iD) 0000-0003-3794-9239

Anabela Rodrigues (iD) 0000-0001-8818-2141

\section{Correspondence to:}

Patrícia Valério Santos

Nephrology Department, Centro Hospitalar de Setúbal

Rua Camilo Castelo Branco, 175, 2910-549 Setúbal, Portugal

Telephone: +351265549555

E-mail: p.valerios89@gmail.com 